\title{
Spatial frequency thresholds for face recognition when comparison faces are filtered and unfiltered
}

\author{
CHARLES A. COLLIN, MEGAN THERRIEN, and CHERON MARTIN \\ University of Ottawa, Ottawa, Ontario, Canada \\ and \\ STEPHANE RAINVILLE \\ York University, Toronto, Ontario, Canada
}

\begin{abstract}
Previous work has shown an advantage of middle spatial frequencies (SFs) in face recognition. However, a few recent studies have suggested that this advantage is reduced when comparison and test stimuli are spatially filtered in a similar way. In the present study, we used standard psychophysical methods, in combination with a match-to-sample task, to determine the SF thresholds for face matching under conditions in which: (1) comparison stimuli were unfiltered and (2) comparison stimuli were spatially filtered in the same way as test stimuli. In two experiments, we show that SFs closer to the middle band are sought out more in the former case than in the latter. These results are compatible with the idea that a middle band of SFs will be most useful for any visual task and that the breadth of this optimal middle band will vary depending on task characteristics.
\end{abstract}

A number of studies have presented evidence that middle spatial frequencies (SFs), between about 8 and 16 cycles per face width $(\mathrm{c} / \mathrm{fw})$, provide information that is more useful for face recognition than are other frequency ranges (Bachmann, 1991; Costen, Parker, \& Craw, 1994, 1996; Gold, Bennett, \& Sekuler, 1999; Näsänen, 1999; Parker \& Costen, 2001; Rolls, Baylis, \& Hasselmo, 1987). On the basis of these data, it has been suggested that band-pass spatial filtering may be the first stage of feature extraction during visual recognition (Näsänen, 1999). That is, the visual information reaching purported face recognition areas might be filtered to preferentially represent middle SFs. In the extreme, it is possible that visual information reaching face recognition areas of the brain is first reduced to only middle SF ranges prior to further analysis. In theory, this filtering could help to minimize the processing load placed on face recognition mechanisms by discarding low and high SFs.

A few recent studies have cast doubt on the idea of an absolute advantage for middle SFs in face recognition by showing that recognition performance is little affected by the range of frequencies contained in face stimuli, so long as both comparison and test stimuli are filtered in the same way (Collin, Liu, Troje, McMullen, \& Chaudhuri, 2004; Kornowski \& Petersik, 2003; Liu, Collin, Rainville, \& Chaudhuri, 2000). These findings seem incompatible with

This work was supported by grants to C.A.C. and M.T. from the Natural Sciences and Engineering Research Council of Canada. We thank Réjean Baribeau of the National Research Council of Canada for his help in calibrating the monitor. Correspondence regarding this article should be addressed to C. A. Collin, School of Psychology, University of Ottawa, Ottawa, ON, K1N 6N5 Canada (e-mail: ccollin@uottawa.ca). the idea that middle SFs provide the most useful information for face recognition. If this were the case, one would expect some degree of performance loss when attempting to match two faces that contain no SFs from inside the middle band, relative to matching two faces with SFs from within the middle band. However, this difference has not been observed. Instead, Collin and colleagues (Collin et al., 2004) have suggested that a broad range of SFs may be useful for any visual task and that the range will depend on such task characteristics as similarity between learned and tested stimuli. This is a view echoed by Schyns and colleagues (Schyns, 1998; Schyns \& Gosselin, 2003), who suggested that a wide range of SFs may be used in any given task and that there is a top-down control of which SFs are accessed in an image.

In the following, we first will discuss two sets of studies that respectively provide evidence for and against the idea of a middle-band advantage in face recognition. We then will present a pair of experiments that examined how the differing task demands of these two sets of studies might lead to different conclusions about the role of SFs in face recognition.

Rolls and colleagues (Rolls et al., 1987) provided some of the earliest evidence that face recognition might rely preferentially on middle SFs. They recorded activity of face-sensitive neurons in the macaque superior sulcus in response to band-pass filtered face images. They found that these neurons responded most strongly to images containing frequencies between 4 and $32 \mathrm{c} / \mathrm{fw}$.

Bachmann (1991) provided the first clue that these physiological effects in monkeys might be directly reflected in human performance. He had participants attempt to recognize images of faces that had been coarse quantized (i.e., pixelated) to different degrees. His data 
showed that recognition performance dropped off sharply as the number of pixels per face width was lowered from 18 to 15 . This finding suggested that SFs below about $8 \mathrm{c} / \mathrm{fw}$ were not sufficient for face recognition. Costen, Parker, and colleagues (Costen et al., 1994, 1996; Parker \& Costen, 2001) followed up on Bachmann's findings, using face images that had been spatially filtered. They found that performance dropped sharply as faces were low-pass filtered below about $8 \mathrm{c} / \mathrm{fw}$ or high-pass filtered above about $16 \mathrm{c} / \mathrm{fw}$. Thus, their findings supported the idea that a middle band of relative SFs was especially useful for face recognition.

Näsänen (1999) added to the evidence for a middle band advantage in a study comparing human performance with that of a computer-based ideal observer algorithm. Ideal observers make judgments based on an optimal use of the objectively available information in images. By factoring out such an algorithm's performance from that of human observers, one obtains a measure of the efficiency with which humans perform the task. Näsänen completed four experiments that showed that human efficiency, relative to that of an ideal observer, was highest when the available SFs were in the middle range (around $8-11 \mathrm{c} / \mathrm{fw}$ ).

In summary, there is evidence from a variety of paradigms supporting a middle-band advantage when test faces are unfiltered and must be matched to filtered comparison faces. However, a few recent studies have suggested that this middle-band advantage is not observed when comparison and test faces are filtered in the same way. Liu and colleagues (Liu et al., 2000) were the first to show this. In the control conditions of their experiments, participants were asked to match pairs of faces that had been filtered in the same way- that is, both low-passed or both high-passed at the same SF cutoff. In these conditions, the results showed no advantage of middle frequencies. Indeed, the participants were able to complete the task with equal accuracy, using a wide range of SFs both inside and outside the middle band of $8-16 \mathrm{c} / \mathrm{fw}$. Similar findings were obtained with a learn/test paradigm.

Although Liu et al.'s (2000) findings were interesting, their studies' implications were limited by the fact that the face images they used during learning and test sessions were in the same view. Because of this, it was possible that their results reflected performance at pattern matching in general and did not have anything to do with face perception per se. Collin and colleagues (Collin et al., 2004) followed up on Liu et al., using faces seen in different views at learn and test, as well as using band-pass filters to eliminate confounding total bandwidth with position on the SF spectrum. The results were identical: When the participants matched face images that were filtered in the same way, no advantage of the middle band emerged.

Most recently, Kornowski and Petersik (2003) have also provided evidence that the accuracy of face recognition varies primarily on the basis of the degree of congruency between comparison and test stimuli, rather than any particular range of SFs that might be included in the stimuli. They had participants learn faces that were high-passed, low-passed, or unfiltered and then had them recognize high-passed, low-passed, and unfiltered faces. Their results showed that accuracy was highest when comparison and test stimuli were similar in SF range (i.e., both highpassed or both low-passed). When comparison faces were unfiltered, accuracy was about equal, regardless of the SF filtering of the test stimuli. Kornowski and Petersik concluded that the overlap in SF between two images is a more important determinant of recognition performance than is the particular ranges of SFs included in it.

In summary, we have briefly reviewed two sets of studies, one showing an advantage of middle SFs and another not. One way to reconcile the findings of these studies is to postulate a visual system that is flexible in its use of SF information (Schyns, 1998; Schyns \& Gosselin, 2003). It may be that rather than relying on a fixed order of processing and rather than preferentially using a given range of SFs for a given class of stimuli, the visual system is versatile enough that a wide range of SFs can be used depending on task characteristics. If this is the case, it should be possible to demonstrate situations under which a broader or a narrower range of SFs is sought out for a given task. The two sets of studies discussed above - those respectively supporting and not supporting a middle-band advantage in face recognition - seem to do this, but the conditions they used have never been compared directly.

One consistent difference between the experiments that support or do not support the middle-band hypothesis is that in one set (those supportive of a middle-band advantage) comparison stimuli are unfiltered, whereas in the second set (those not supportive of a middle-band advantage) comparison stimuli are filtered in the same way as test stimuli. To our knowledge, only Kornowski and Petersik (2003) have examined in detail conditions in which comparison stimuli are both filtered and unfiltered (but see Gold et al., 1999, p. 3551). However, because they examined only low-passed and high-passed conditions, their results do not bear directly on the question of whether middle frequencies might be advantageous in cases in which comparison stimuli are unfiltered. In the following, we will present two experiments that directly compare the conditions used in experiments that support a middle-band advantage and those that do not. That is, we directly compare conditions in which comparison stimuli are filtered versus those in which they are unfiltered. If frequencies closer to the middle band are sought out when comparison faces are unfiltered, relative to when they are filtered, this forces a modification of ideas concerning the optimality of middle SFs in face recognition and favors the view that there is a high degree of flexibility in how the visual system uses SF information (Schyns, 1998; Schyns \& Gosselin, 2003).

One difficulty with previous studies that have shown a lack of a middle-band advantage is that their conclusions necessarily relied on null results (Collin et al., 2004; Kornowski \& Petersik, 2003; Liu et al., 2000). That is, they relied on a statistical result where the null hypothesis failed to be rejected. The difficulty of interpreting such results is well known. In the experiments that follow, we endeavored to improve on this past work by examining similar 
questions in such a way that a positive result showing a lack of a need for middle-band frequencies was possible.

Another weakness of past studies in this area-this one applying to both those that show a middle-band advantage and those that do not - is that they have universally examined SFs by looking at recognition performance as a function of a few discrete and isolated ranges of frequencies. The limits of the critical band for face recognition have, therefore, had to be inferred by interpolating between performance levels at these different isolated points. For example, in Costen et al.'s (1996) Experiment 2, face images were high-pass filtered at 4.5, 6, 11.5, and $22.5 \mathrm{c} / \mathrm{fw}$, and performance on these four conditions was measured. A drop in performance on the $22.5-\mathrm{c} / \mathrm{fw}$ condition, relative to the other three, was observed. It was therefore estimated that a threshold of useful SFs for face recognition existed somewhere between 11.5 and $22.5 \mathrm{c} / \mathrm{fw}$, but due to the highly discrete way in which SF was treated, only a fairly rough estimate of the limits could be obtained. All other studies in this area of which we are aware used this same general approach. Essentially, the weakness of this approach is that it examines SF in very coarse units. In the experiments that follow, we used the psychophysical methods of adjustment and constant stimuli, in combination with a match-to-sample procedure, to obtain more precise SF thresholds for face recognition. In doing this, we were able to treat SF in a more continuous manner, and we were thus able to analyze the SF ranges necessary for recognition in a more exact way than in previous studies.

\section{EXPERIMENT 1}

In Experiment 1, we used the method of adjustment to determine the SF thresholds for face recognition under conditions in which comparison faces were either filtered or unfiltered. It was anticipated that the participants would seek out middle SFs when performing the task under conditions in which the comparison faces were unfiltered while the test face was filtered, but not when both comparison and test faces were filtered in the same manner. This would be compatible with the notion of a flexible, rather than fixed, use of SF ranges by the visual system.

\section{Method}

Participants. Twenty undergraduate students ( 7 of them male) from the University of Ottawa participated for a small honorarium. The mean age of the participants was $23.0 \pm 6.4$ years. All had normal or corrected-to-normal vision.

Stimuli and Materials. The stimuli consisted of images of 27 individual 3-D face models from the Max Planck Institute for Biological Cybernetics Face Database (faces.kyb.tuebingen.mpg.de/). Seven of these faces ( 3 of them male) were reserved for practice trials. The remaining 20 (10 of them male) were used for experimental trials. All the faces were imaged in two views: face-on $\left(0^{\circ}\right)$ and three-quarter view $\left(22.5^{\circ}\right)$. Each face image was reduced to a $256 \times 256$ pixel 8-bit grayscale picture, with the face stimulus itself

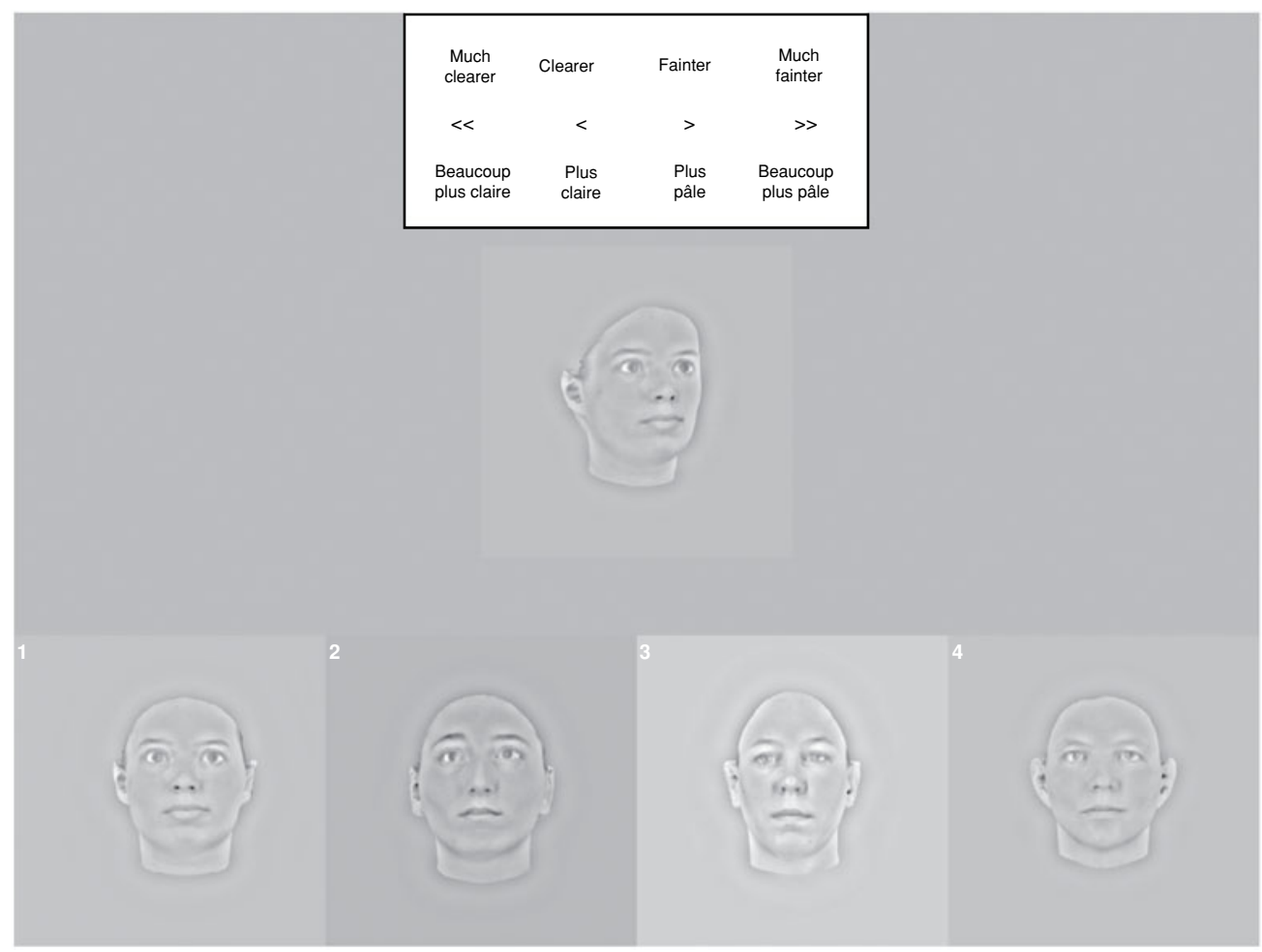

Figure 1. Example of the screen's appearance in the filtered comparison faces condition. Arrows at the top of the screen indicate the effects of the keys used to adjust the spatial frequency cutoff of the target and comparison faces. In this case, both test and comparison faces are filtered in the same way. 
being 128 pixels wide and surrounded by a gray background. The SF filters applied to the face images were circularly symmetric Butterworth filters of exponent 5. Prior to filtering, all the faces were equated for mean luminance and RMS contrast. Following filtering, the faces were returned to the same mean luminance that they had prior to filtering (i.e., the DC component of the original was substituted for that of the filtered version). Example stimuli can be seen in Figures 1 and 2.

The experiment was run using MATLAB 5.2 (The Mathworks, Inc., Natick, MA) on a Macintosh Dual 2-GHz PowerPC G5. The stimuli were presented on an NEC AccuSync 95F 17-in. monitor. The monitor was properly calibrated for linear luminance, using a Minolta CS-1000 photometer (Minolta Canada, ON, Canada). A chinrest was used to maintain a fixed viewing distance.

Procedure. The experiment employed a $2 \times 2$ fully factorial mixed design, with the factors being filter type (low-pass or highpass, within subjects) and comparison face filtering (unfiltered or filtered, between subjects). Note that low-pass and high-pass levels here refer to the filtering of only the test face in the case of unfiltered comparison faces condition. In all the conditions, the psychophysical method of adjustment was used in combination with a match-tosample recognition task. Further details will be given below.

To begin the experiment, the participants were seated at a viewing distance of $57 \mathrm{~cm}$ from the computer monitor, so that $1 \mathrm{~cm}$ on the screen subtended $1^{\circ}$ of visual arc. They were first presented with the instructions for the experiment and then did 4 practice trials, after which they were presented with the instructions a second time to ensure that they understood the task before starting the experimental session. The experimental session consisted of 80 trials. Total participation time was approximately $1 \mathrm{~h}$.

On each trial of the experiment, the participants were presented with a test face in the middle of the screen and four comparison faces along the bottom of the screen that were numbered from 1 to 4. The participants were instructed to adjust the clarity of the test face (concomitantly with the comparison faces if they were in the filtered comparison faces condition) to the point where it could just be recognized as one of the comparison faces. That is, the participants were asked to adjust the SF cutoff of the test face (again, along with comparison faces if in the filtered comparison faces condition) to their recognition threshold. On half the trials, high-pass filter was applied to the face(s), and on the other half, a low-pass filter was applied to the face(s). Within each of these filter type conditions, half the trials involved increasing the clarity of an initially unclear image - that is, one that was extremely low-passed (cutoff, $1 \mathrm{c} / \mathrm{fw}$ ) or extremely high-passed (cutoff, $76 \mathrm{c} / \mathrm{fw}$ ). The other half of the trials involved decreasing the clarity of an initially clear image - that is, one that began only very slightly low-passed (cutoff, $76 \mathrm{c} / \mathrm{fw}$ ) or very slightly high-passed (cutoff, $1 \mathrm{c} / \mathrm{fw}$ ). This is in keeping with the standard psychophysical method of adjustment, in which the trials are typically divided evenly among ascending trials, on which observers increase a stimulus's intensity, and descending trials, on which observers decrease a stimulus's intensity.

The participants were able to adjust the filtering cutoff as desired, so that they might, for instance, increase the clarity of an initially unclear image to an extreme degree before reducing it back to what they felt was their recognition threshold. They were able to vary the cutoff up and down several times as they attempted to find their threshold. No limits were placed on this aspect of the task, and the participants were encouraged to explore the range around their subjective threshold.

Half of the participants performed the task in a condition in which only the test face was spatially filtered (the unfiltered comparison faces condition). For the other half of the participants, both the test and the comparison faces were filtered in the same way and changed

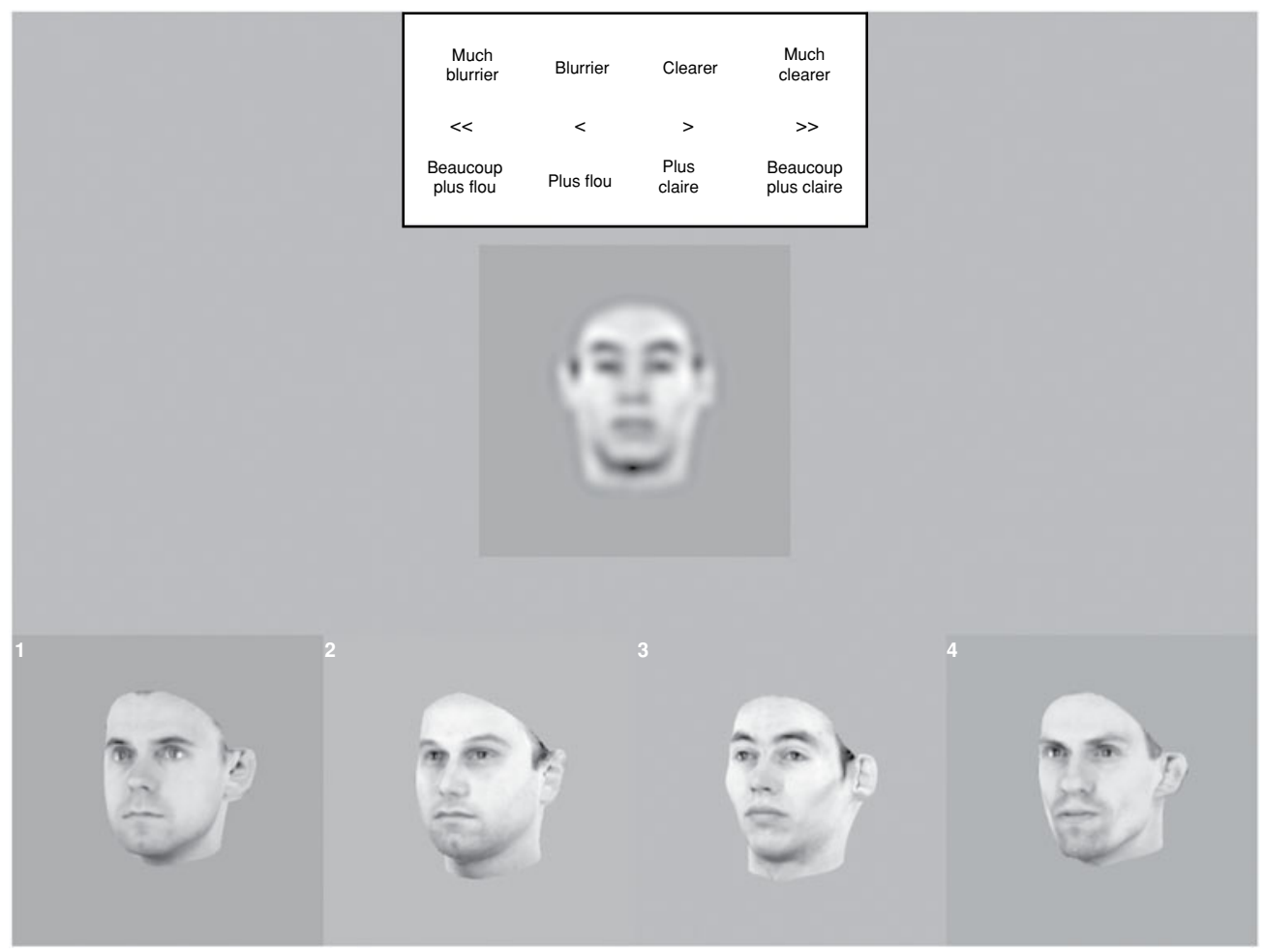

Figure 2. Example of screen's appearance during the experiment. At the top of the screen, arrows indicate the effects of the keys used to adjust the spatial frequency cutoff of the target face, which appears in the center of the screen and is somewhat low-passed in this example. Comparison faces appear at the bottom of the screen. In this case, they are unfiltered, since they appeared in the unfiltered comparison faces condition. 
together as the participant adjusted the threshold of the SF filter (the filtered comparison faces condition).

In the practice trials, the participants completed 2 trials with high-pass filtered faces and 2 with low-pass filtered faces. In the experimental session, 40 trials from each condition were completed. The conditions were fully blocked, and the blocks were presented in random order.

The participants made their adjustments of the SF cutoffs with four keys, marked with a double left arrow $(<<)$, a single left arrow $(<)$, a single right arrow $(>)$, or a double right arrow $(>>)$, respectively. The left arrows lowered the SF cutoff of the filter, whereas the right arrows increased it. Single arrow keys made an adjustment of 0.1 octaves of SF, whereas double arrows yielded adjustments of 0.5 octaves. Instructions on the screen constantly indicated the effects of the arrow keys on the appearance of the image, so as to avoid participant confusion (see the tops of Figures 1 and 2). Once the recognition threshold was found, a participant indicated which of the four comparison faces matched the test face, using keyboard keys numbered 1 through 4 .

Each of the 20 faces was used twice per block as a test face-once in an ascending trial, and once in a descending trial. The faces were presented in random order, with the constraint that the same face could not appear on successive trials. On each trial, three foils were selected randomly from among the 9 other faces of the same gender and were placed, along with the correct face, at the bottom of the screen. The positions of the correct face and the foils along the bottom of the screen were randomly assigned on a trial-by-trial basis. On half of the trials in each block, the test face was shown face-on, and the comparison faces were shown in three-quarter view. On the other half of the trials, the test face was shown in three-quarter view, and the comparison faces were shown face-on. Thus, the faces were always being matched across a $22.5^{\circ}$ difference in angle of view.

\section{Results}

The results of the experiment are demonstrated in Figure 3, which shows the mean SF thresholds and standard errors. Threshold here refers to the point on the SF spectrum to which the participants adjusted the $50 \%$ cutoff point of the high-pass or low-pass filter. As is typical with the method of adjustment, the threshold for each condition was obtained by averaging across ascending and descending trials. The differences in threshold between ascending and descending trials were small, approximately 0.2 octaves. Accuracy in all the conditions was above $95 \%$, as was anticipated, and was not further analyzed.

As can be seen in Figure 3, the main finding in this experiment is that the participants in the unfiltered comparison faces condition consistently adjusted the cutoff point of the low-pass and high-pass filters to points closer to middle frequencies than did those in the filtered comparison faces condition. Indeed, those in the unfiltered comparison faces condition adjusted thresholds to points within the $8-16 \mathrm{c} / \mathrm{fw}$ range suggested as the critical middle band by previous studies (Costen et al., 1994, 1996; Parker \& Costen, 2001), whereas those in the latter group adjusted the thresholds to points outside this critical band.

The pattern of results was confirmed by a $2 \times 2$ mixed ANOVA with filter type (low-pass or high-pass, within subjects) and comparison faces (filtered or unfiltered, between subjects) as factors. This showed a significant interaction of the two factors $[F(1,20)=18.117, p=.0004]$. There was also a main effect of filter type $[F(1,20)=$ $55.433, p<.0001]$; however, the overall effect in the comparison face condition was not significant $[F(1,20)<1]$. Examination of the simple main effects in the comparison faces condition within each level of the filter type variable showed that the threshold for high-pass filtering was significantly higher when the comparison faces were filtered than when they were unfiltered $[t(20)=3.050$, $p=.0063]$, whereas the threshold for low-pass filtering was significantly lower when the comparison faces were filtered than when they were unfiltered $[t(20)=-4.60$, $p=.0002]$. Specifically, the mean high-pass thresholds were 20.11 and $13.99 \mathrm{c} / \mathrm{fw}$ for the filtered and unfiltered comparison faces conditions, respectively, whereas the low-pass thresholds were 6.50 and $10.56 \mathrm{c} / \mathrm{fw}$ for the

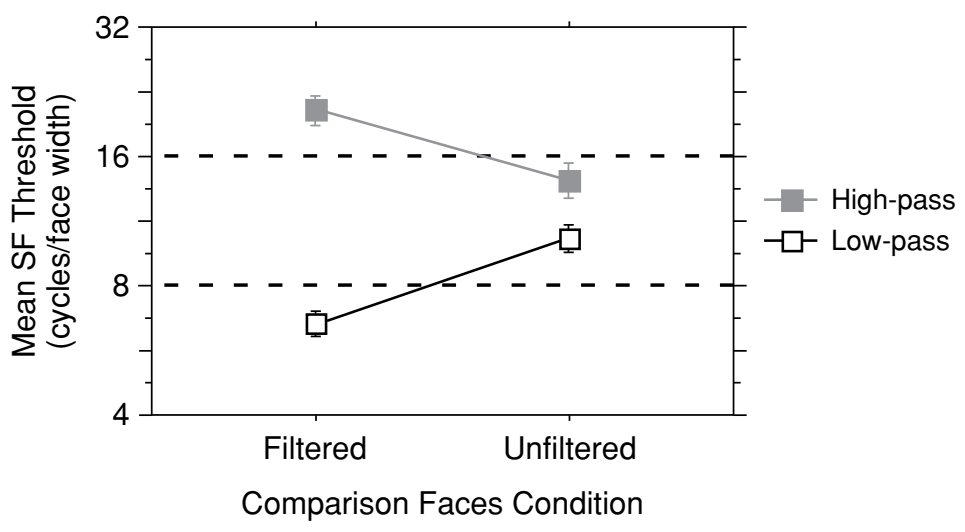

Figure 3. Results for Experiment 1. Mean low-pass and high-pass thresholds obtained by the method of adjustment for face recognition (match to sample) under conditions in which comparison faces were either unfiltered or filtered in the same way as the test face. The dotted lines indicate the range of the critical middle spatial frequency (SF) band for face recognition, as suggested by previous research. The error bars represent one standard error of the mean threshold. 
filtered and unfiltered comparison faces conditions, respectively. In short, the thresholds were more extreme, and outside the middle band as it is usually defined, when the comparison faces were filtered in the same way as the test face, whereas they were inside the middle band when a filtered test face was being compared with unfiltered comparison faces.

Our findings also provide a direct quantitative estimate of the range of SFs useful for face recognition under two different testing conditions. When filtered test faces were matched to unfiltered comparison faces, thresholds of 10.56 and $13.99 \mathrm{c} / \mathrm{fw}$ were obtained for low-pass and high-pass filtered, respectively. When test faces were matched to comparison faces that had been similarly filtered, thresholds of 6.50 and $20.11 \mathrm{c} / \mathrm{fw}$ were obtained for low-pass and high-pass, respectively. Thus, the range of useful frequencies when two similarly filtered faces are matched is about an octave wider than when unfiltered faces are matched to filtered ones.

On the basis of the threshold values above, and assuming that a 2-octave-wide band of frequencies is needed for recognition (Gold et al., 1999; Näsänen, 1999), we can estimate that the range of useful frequencies is about $5.28-27.98 \mathrm{c} /$ $\mathrm{fw}$ when test faces are unfiltered and about $3.25-40.22 \mathrm{c} /$ fw when both are filtered at the same cutoff. These values were calculated by subtracting 1 octave from the low-pass threshold and adding 1 octave to the measured high-pass threshold. That is, we have assumed in these calculations that the participants were using a 2-octave-wide band exactly centered on the threshold values. This assumption is admittedly somewhat arbitrary, but it does yield estimates of useful frequency thresholds that are in agreement with previous work; for example, Rolls et al.'s (1987) estimate of 4-32 c/fw corresponds well with our estimate for matching unfiltered faces to filtered ones.

\section{Discussion}

The results of this experiment showed that the participants sought out middle-band SFs when performing a task that required them to match a spatially filtered face image with an unfiltered one but that the participants could perform the same task with more extreme frequencies when matching similarly filtered faces. These data are compatible with those in previous studies (Collin et al., 2004; Liu et al., 2000) showing that middle SFs do not have an advantage when test and comparison stimuli are similarly filtered, as well as with those in studies showing an advantage of the middle band with comparison stimuli that are unfiltered (Bachmann, 1991; Costen et al., 1994, 1996; Gold et al., 1999; Näsänen, 1999; Parker \& Costen, 2001; Rolls et al., 1987). In Experiment 1, we have provided data that directly compare the two situations previously explored separately in these two groups of studies and have found a positive result, indicating that the difference between their findings is indeed due to differences in the congruency of learn and test stimuli. This suggests that a different range of SFs is used by the visual system, depending on the type of face recognition task being engaged in.
One weakness of the results above is that they rely on the method of adjustment. Although this method is straightforward and quick to execute, it is susceptible to criterion shifts. That is, participants making adjustments in different conditions might exhibit different degrees of desired certainty prior to making responses. This would affect the threshold values assessed from their responses. In Experiment 1, it is possible that the participants in the unfiltered condition were exhibiting a more stringent matching criterion than were those in the filtered comparison condition, perhaps because the latter condition was perceived as easier in some sense. This would have caused their thresholds to shift toward the middle. This produces an alternative explanation for our results. To test this interpretation of the results, in Experiment 2 we attempted to replicate Experiment 1, but using the method of constant stimuli, which is less vulnerable to criterion shifts.

\section{EXPERIMENT 2}

Experiment 2 replicated Experiment 1, using the method of constant stimuli. As with Experiment 1, the goal was to determine the SF thresholds for face recognition under conditions in which comparison faces were either filtered or unfiltered.

\section{Method}

Participants. Thirty-seven undergraduate students ( 5 of them male) from the University of Ottawa participated for a small honorarium or course credit. The mean age of the participants was $21.43 \pm 4.16$ years. All had normal or corrected-to-normal vision.

Stimuli and Materials. The stimuli and materials were the same for Experiment 2 as for Experiment 1, except that, in this case, there were 10 test faces ( 5 of them male) and 7 practice faces ( 3 of them male). For this experiment, spatial filtering was applied to each face image to produce 18 filtered band-passed versions. The band-pass filters were 2 octaves wide. There were 9 high-pass filters (i.e., bandpass filters in the high end of the spectrum) with center frequencies equally spaced 0.25 octaves apart from 19.03 to $76.11 \mathrm{c} / \mathrm{fw}$ and, thus, high-pass cutoffs of 9.51-38.06 c/fw. There were also 9 lowpass filters (i.e., band-pass filters in the low end of the spectrum) with center frequencies equally spaced 0.4 octaves apart from 1.32 to $12.13 \mathrm{c} / \mathrm{fw}$ and, thus, low-pass cutoffs of $2.64-24.26 \mathrm{c} / \mathrm{fw}$. These values were chosen on the basis of the results of Experiment 1 and pilot testing with the method of constant stimuli.

As in Experiment 1, the SF filters applied to the face images were circularly symmetric Butterworth filters of exponent 5 . Prior to filtering, all the faces were equated for mean luminance and RMS contrast. Following filtering, the faces were returned to the same mean luminance that they had prior to filtering (i.e., the DC component of the original was substituted for that of the filtered version).

Procedure. The procedure in Experiment 2 was very similar to that in Experiment 1, except that the method of constant stimuli was employed to determine thresholds for face recognition, instead of the method of adjustment, and the design was fully within subjects, instead of being mixed. These and other minor differences are detailed below.

Experiment 2 employed a $2 \times 2$ fully factorial, fully withinsubjects design, with factors being filter type (low-pass or highpass) and comparison face filtering (unfiltered or filtered). In all the conditions, the psychophysical method of constant stimuli was used in combination with a $4 \mathrm{AFC}$ match-to-sample recognition task. As in Experiment 1, the participants were seated at a viewing distance of $57 \mathrm{~cm}$ from the computer monitor; they were then presented with 
the instructions for the experiment, followed by 14 practice trials, after which they were presented with the instructions a second time to ensure that they understood the task before starting the experimental session.

The experimental session consisted of 720 trials. This broke down into 9 filtering levels $\times 2$ filtering types (high-pass or low-pass) $\times$ 2 comparison face filtering conditions (filtered or unfiltered) $\times 20$ trials per condition per filter. The conditions were blocked across the comparison filtering condition, and the order of the levels of this factor were counterbalanced across participants. All other factors were unblocked, and the trials were presented in random order. Total participation time was approximately $1 \mathrm{~h}$.

On each trial of the experiment, the participants were presented with a test face in the middle of the screen and four comparison faces along the bottom of the screen that were numbered 1 through 4 . In the unfiltered comparison faces condition, the face in the center of the screen was band-pass filtered to one of the 18 filtering levels described above, and the comparison faces were unfiltered. In the filtered comparison faces condition, all the faces (test and comparison) were filtered with the same band-pass filter. The participant's task was to choose which of the comparison faces matched the test face.

On half of the trials in each block, the test face was shown face-on, and the comparison faces were shown in three-quarter view. On the other half of the trials, the test face was shown in three-quarter view, and the comparison faces were shown face-on. Thus, the faces were always being matched across a $22.5^{\circ}$ difference in angle of view.

\section{Results}

The results of the experiment are shown in Figure 4, which shows the mean SF thresholds and standard errors. Thresholds for each participant were calculated by fitting a cumulative $\log (x)$ normal function to their accuracy results across the nine filtering levels in each condition. Because we used a $4 \mathrm{AFC}$ task, performance ranged from $25 \%$ to $100 \%$ correct. Thresholds were calculated at a $62.5 \%$ accuracy level, halfway between chance-level performance and maximum performance.

As can be seen in Figure 4, the main finding in this experiment is similar to that in Experiment 1: The par- ticipants consistently showed low-pass and high-pass thresholds closer to the middle frequencies when comparison faces were unfiltered than they did in the filtered comparison condition, although the difference with highpass faces was quite small, in comparison with Experiment 1 . In the low-pass condition with unfiltered comparison faces, thresholds were adjusted to points within the $8-16 \mathrm{c} / \mathrm{fw}$ range suggested as the critical middle band by previous studies (Costen et al., 1994, 1996; Parker \& Costen, 2001), whereas in the low-pass condition with filtered comparison faces, the thresholds were adjusted to points outside this critical band. In both high-pass conditions (i.e., with filtered and unfiltered comparison faces), the thresholds were somewhat elevated, relative to those in previous studies, but values for the filtered comparison condition were, nonetheless, slightly closer to the middle band than were values from the unfiltered condition, providing a qualitative agreement with Experiment 1.

The visible pattern of results was confirmed by a $2 \times 2$ repeated measures ANOVA with filter type (low-pass or high-pass) and comparison faces filtering (filtered or unfiltered) as factors. This showed a significant interaction of the two factors $[F(1,36)=16.812, p=.0002]$. There was also a main effect of filter type $[F(1,36)=55.433$, $p<.0001]$. The overall effect of comparison filtering was not significant $[F(1,36)=3.175, p=.0832]$. Examination of the simple main effects of the comparison faces factor within each level of the filter type variable showed that the threshold for high-pass filtering was significantly higher when the comparison faces were filtered than when they were unfiltered $[t(36)=2.048, p=.0479]$, whereas the threshold for low-pass filtering was significantly lower with filtered comparison faces than with unfiltered ones $[t(36)=-4.701, p<.0001]$. This is the same pattern of results as that found in Experiment 1, although again, the effect for the high-pass faces was very small in magnitude.

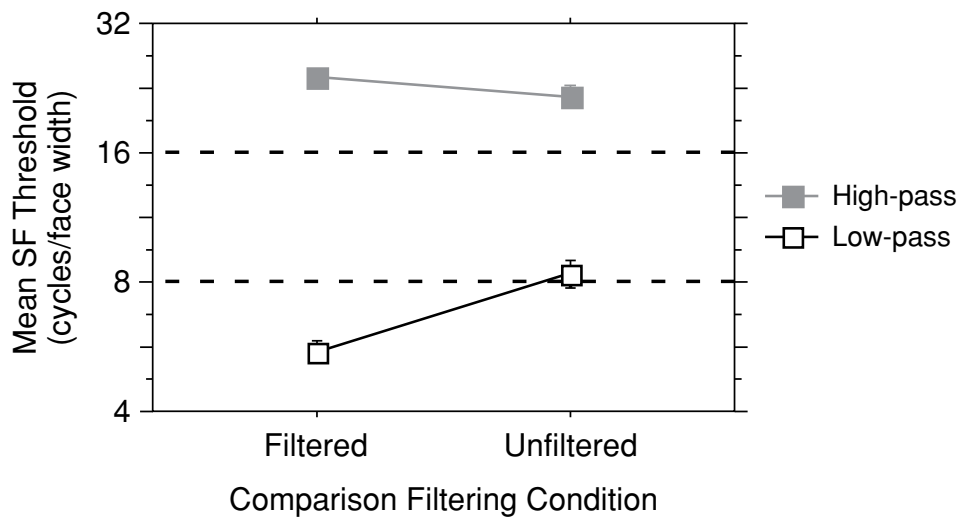

Figure 4. Results for Experiment 2. Mean low-pass and high-pass thresholds obtained by method of constant stimuli for face recognition (match to sample) under conditions in which comparison faces were either unfiltered or filtered in the same way as the test face. The dotted lines indicate the range of the critical middle spatial frequency (SF) band for face recognition, as suggested by previous research. The error bars represent one standard error of the mean threshold. 
The mean high-pass thresholds were 24.51 and $22.72 \mathrm{c} / \mathrm{fw}$ for the unfiltered and filtered comparison faces conditions, respectively, whereas the low-pass thresholds were 5.78 and $9.33 \mathrm{c} / \mathrm{fw}$ for the unfiltered and filtered comparison faces conditions, respectively. In short, both low-pass and high-pass thresholds were more extreme when comparison faces were filtered in the same way as the test face, whereas they were closer to the middle band when a filtered test face was being compared with unfiltered comparison faces.

As in Experiment 1, the findings of Experiment 2 can provide a quantitative estimate of the range of SFs useful for face recognition under two circumstances. When filtered test faces were matched to unfiltered comparison faces, thresholds of 9.33 and $22.72 \mathrm{c} / \mathrm{fw}$ were obtained for low-pass and high-pass filtered, respectively. When test and comparison faces that had been similarly filtered were matched, thresholds of 5.78 and $24.51 \mathrm{c} / \mathrm{fw}$ were obtained for low-pass and high-pass, respectively. Thus, the range of useful frequencies when two similarly filtered faces are matched is about 1 octave wider than when unfiltered faces are matched to filtered ones. As in Experiment 1, the threshold values above can be used to derive an estimate of the range of useful frequencies for face recognition. Assuming a 2-octave-wide band of frequencies is needed for recognition (Gold et al., 1999; Näsänen, 1999), we estimate that the range of useful frequencies is about $4.66-45.44 \mathrm{c} / \mathrm{fw}$ when test faces are unfiltered and about $2.89-49.02 \mathrm{c} / \mathrm{fw}$ when both test and comparison faces are filtered at the same cutoff. See the Results section for Experiment 1 for more on the derivation of these values.

\section{Discussion}

The results of Experiment 2 generally replicated those of Experiment 1. We found in both experiments that thresholds for filtered comparison images were more extreme than those in the unfiltered comparison images condition. As in Experiment 1, these data are compatible with those in previous studies (Collin et al., 2004; Liu et al., 2000), showing that middle SFs do not have an advantage when test and comparison stimuli are similarly filtered, as well as with those in studies showing an advantage of the middle band with comparison stimuli that are unfiltered (Bachmann, 1991; Costen et al., 1994, 1996; Gold et al., 1999; Näsänen, 1999; Parker \& Costen, 2001; Rolls et al., 1987).

The only notable difference in results between Experiments 1 and 2 is that the high-pass conditions in Experiment 2 seem to have elicited more extreme thresholds overall and the difference between filtered and unfiltered conditions here is much smaller. The data show the same general pattern in the two experiments, but there are quantitative differences for the high-pass condition. It is possible that some of the difference between the filtered and the unfiltered comparison faces conditions with high-pass images in Experiment 1 was due to a criterion shift, but the results of Experiment 2 show that such a criterion shift cannot account for the entire effect. Interestingly, the highpass threshold is outside the middle band for both filtered and unfiltered comparison faces conditions, suggesting that the upper limit of the middle band may have been underestimated in some previous studies (including Experiment 1). However, it should be noted that some previous work has shown an upper limit in the range of 20-25 c/fw, compatible with our results (T. Hayes, Morrone, \& Burr, 1986; Näsänen, 1999).

\section{GENERAL DISCUSSION}

The results of the two experiments reported in this article showed that the participants used middle-band SFs when doing a task that required them to match a spatially filtered face image with an unfiltered one but that the participants could perform the same task with more extreme frequencies when matching similarly filtered faces. These data are not compatible with the idea of a fixed use of a given spatial frequency range for face recognition and, instead, favor the theory that the visual system is flexible in its use of SFs (Schyns, 1998; Schyns \& Gosselin, 2003).

Our data are compatible with those in previous studies (Collin et al., 2004; Liu et al., 2000) showing that middle SFs do not have an advantage when test and comparison stimuli are similarly filtered, as well as with those in studies showing an advantage of the middle band with comparison stimuli that are unfiltered (Bachmann, 1991; Costen et al., 1994, 1996; Gold et al., 1999; Näsänen, 1999; Parker \& Costen, 2001; Rolls et al., 1987). We have provided data that directly compare the two situations previously explored separately in these groups of studies and have found a positive result, indicating that the difference between their results is, indeed, due to differences in the congruency of comparison and test stimuli. We have thus helped to reconcile the findings of these two groups of past studies.

Our findings also provide a direct quantitative analysis of the range of frequencies useful for face recognition. We found that when filtered test faces are matched to filtered comparison faces, the range of useful frequencies is about an octave wider than when filtered test faces are matched to unfiltered comparison faces. This again serves to reconcile studies that have shown evidence for and against a middle-band superiority. Those showing no evidence of a middle-band advantage have likely done so due to the wider range of frequencies useful for matching similarly filtered images.

The precise range of frequencies useful for matching unfiltered faces to filtered faces is somewhat wider as calculated in our study (5.12-27.98 c/fw in Experiment 1, 4.66-45.55 c/fw in Experiment 2) than has been suggested by previous ones (e.g., $8-16 \mathrm{c} / \mathrm{fw}$ suggested by Costen et al., 1996). It should be noted, however, that our results match fairly well with those of Rolls and colleagues (Rolls et al., 1987), who showed that face neurons in the macaque respond to frequencies between 4 and $32 \mathrm{c} / \mathrm{fw}$. Although our suggested upper range may seem somewhat elevated on the basis of most of the human behavioral data, our Experiment 1 results are compatible with early findings (A. Hayes, 1988; Näsänen, 1999; Tieger \& Ganz, 1979), 
which suggested that the upper limit of the optimal band of frequencies for face recognition is around $20-25 \mathrm{c} / \mathrm{fw}$.

It is interesting to note that the high SF threshold in Experiment 2 was around $20 \mathrm{c} / \mathrm{fw}$ for both the filtered and the unfiltered comparison face conditions. Unlike in Experiment 1, the unfiltered condition did not yield a threshold within the middle band. Instead, both thresholds were outside the middle band as it has traditionally been defined (Costen et al., 1994, 1996), although the threshold in the unfiltered comparison images conditions was closer to the middle range. This may suggest a middleband upper limit closer to $20 \mathrm{c} / \mathrm{fw}$, again compatible with several previous studies (A. Hayes, 1988; Näsänen, 1999; Tieger \& Ganz, 1979). However, the interpretation of this result is complicated by the fact that the high-pass threshold in Experiment 1 was within the middle band when comparison images were unfiltered. This may reflect the action of criterion shifts allowed by the more subjective method of adjustment used in Experiment 1.

A number of theories have been proposed in previous studies examining face recognition that might explain why a broader range of SFs would be useful in matching a filtered test face to filtered comparison faces than would be useful for matching two similarly filtered face images. Here, we will briefly outline these theories in order to give our findings theoretical context. However, we should note that the present data are not sufficient to provide a clear choice among these hypotheses. Indeed, it may be that several of them are correct in combination and to varying degrees.

Liu et al. (2000) and Collin et al. (2003) have suggested that an analysis of face recognition and SF requires a consideration of the degree of overlap in SF range between comparison and test stimuli. They found that when face images share a wide range of SFs, the position on the spectrum that the frequencies occupied had minimal effects on recognition accuracy. They argued that when one image contains frequencies not present in another, these additional frequency elements interfere with matching one with the other. In this view, the middle SFs may be more useful than other frequency ranges, but the frequency range is not the primary determinant of recognition performance (whether measured in terms of RT, matching accuracy, or recognition accuracy); instead, it is the congruency in SF range between comparison and test stimuli. Thus, when interference by other frequencies is high and overall performance is low - that is, when an unfiltered face is matched to a filtered one - the advantage of the middle band stands out. However, when interference is low and overall performance is high - that is, when two images that are similarly filtered are matched - the middle-band advantage is small and may not be observed.

Boutet and colleagues (Boutet, Collin, \& Faubert, 2003) examined whether the middle-band advantage in faces might be related to the fact that face recognition is based more on configural information than on object recognition (Mauer, Le Grand, \& Mondloch, 2002). They reasoned that if face recognition relied preferentially on middle SFs and if it also relied on configural information, the former might carry the latter. They had participants attempt to recognize face and house images that had been filtered to either include or exclude middle SFs, hypothesizing that removal of middle frequencies would impair upright face recognition but not house recognition. However, they found no evidence that middle frequencies disproportionately carried configural information.

Boutet et al. (2003) suggested, instead, that the middleband advantage might be due to the middle range of frequencies having sufficient amounts of both contrast and spatial information to be useful. The contrast in a natural image drops as $1 / f^{n}$, where $f$ is frequency and $n$ is an exponent in the range of about $1-2$ (Field \& Brady, 1997). This means that the amount of contrast in a natural image is halved for every doubling of frequency. On the other hand, the amount of spatial information carried by a band of frequencies rises as one goes up the spectrum. This is because there are more frequency coefficients in a given octave of higher frequencies than in the same $\log$ bandwidth of lower frequencies. In a manner analogous to having more pixels in the spatial domain, this generally (although not necessarily) provides more possibilities for distinguishing differences between images. It should be made clear that the purported higher level of spatial information in higher SF bands depends on image characteristics. If the high SF coefficients do not vary between images, obviously no distinguishing information is provided by them, and so the greater number of coefficients in the higher ranges will not provide any distinction between images. However, in natural images, it is highly likely that there will be variations in these higher coefficients.

In short, Boutet et al. (2003) suggested that middle SFs may represent a "happy medium" between low frequencies that have a great deal of contrast but little spatial information and high frequencies that have little contrast but more spatial information. In Boutet and colleagues' view, there will be a middle band of SFs that is useful for any given visual task simply because the basic characteristics of extreme SFs make them objectively less informative or less visible, but the extent of the middle band will be highly flexible and will depend on task-specific task characteristics, not just the class of stimuli being used.

Another potential explanation of our results comes from Schyns and colleagues (Morrison \& Schyns, 2001; Schyns, 1998). They have shown that the usefulness of different bands of SFs is task dependent and that there is a degree of top-down control over the ranges of SFs that are accessed first by the visual system when doing scene and face recognition. On the basis of these findings, one might postulate that the differences between the filtered and the unfiltered comparison faces conditions are a result of a top-down selection of the most useful spatial frequency bands for each task. It may be the case that when the full band of frequencies is available at learning, this is selected as the most useful band, and information from that band is preferentially encoded. However, when the middle frequencies are unavailable or only partially available, the available band of frequencies is chosen for encoding in- 
stead. Thus, during the recognition phase of the present experiments, if the full band was available at encoding, superior performance with the middle band was observed, whereas no such advantage was observed when a limited band of frequencies was available at encoding.

In all of the explanations above, it is suggested that the middle band of SFs might be more useful or informative for face recognition. However, it should be noted that the reason why the middle band might be most useful (or at least most used) is, at the moment, an unanswered question. It cannot be that the middle-band has a higher similarity with other bands on a simple point-by-point basis, since any two images containing only isolated bands of SFs have a zero-order pixel-by-pixel correlation by definition. It must, therefore, be some kind of higher order information that is being extracted from the raw image that makes the middle band more useful. One candidate for this higher order information is configural data, but Boutet et al. (2003) have shown that the middle band does not preferentially carry configural information for face recognition. One remaining possibility as to the nature of this higher order information is that it consists of distortion products produced by a nonlinearity applied by the face recognition system to middle-band information. However, although there is support for nonlinearities in the way the visual system deals with spatial and temporal modulations (Burton, 1973; He \& MacLeod, 1996), to our knowledge, there is no empirical evidence to support the idea that they are applied specifically to the middle SF band in face recognition.

A consideration that must be taken into account in analyzing our results is that we used a simultaneous matching paradigm. Although this paradigm has been used previously (Collin et al., 2004; Liu et al., 2000) and provides a well-controlled experimental situation, there are some limitations in generalizing from such a task to real-world face recognition. Most notably, there is negligible memory load involved. It is possible that different results would be obtained in a task requiring greater memory load, such as a delayed match-to-sample paradigm. However, a simultaneous matching task is necessary if one is to use the method of adjustment while comparing filtered and unfiltered comparison images, as was done in Experiment 1. This is because it is obviously not possible to adjust the filtering of a face image once it has been memorized. It is possible to use a delayed match-to-sample technique in the method of constant stimuli, but we decided to use the same basic task in Experiment 2 as in Experiment 1, in order to maintain comparability. In further studies, we will examine results under conditions of greater memory load; however, our previous work suggests that similar SF effects are observed with various memory loads, despite overall changes in performance level (Collin et al., 2004; Liu et al., 2000).

In summary, the present results show a variation in the extent of the middle band of SFs that is sufficient for face recognition. The extent of the band is broader when comparison and test faces are filtered in the same way, in comparison with a situation in which comparison faces are unfiltered whereas test faces are filtered. We provide several specific theories as to why this might be the case, although we note that the data available in the literature does not as yet allow us to select among them. We do note that our results are incompatible with the possibility of band-pass filtering being a mandatory first step in face processing. Instead, our results, along with an analysis of other findings in the literature, suggest that the face recognition process is flexible with regard to the bands of frequencies it employs.

\section{REFERENCES}

BaChmanN, T. (1991). Identification of spatially quantised tachistoscopic images of faces: How many pixels does it take to carry identity? European Journal of Cognitive Psychology, 3, 87-103.

Boutet, I., Collin, C. [A.], \& Faubert, J. (2003). Configural face encoding and spatial frequency information. Perception \& Psychophysics, 65, 1078-1093.

BURTON, G. J. (1973). Evidence for non-linear response processes in the human visual system from measurements on the thresholds of spatial beat frequencies. Vision Research, 13, 1211-1225.

Collin, C., Liu, C. H., Troje, N., McMullen, P. A., \& Chaudhuri, A. (2004). Face recognition is affected by similarity in spatial frequency range to a greater degree than within-category object recognition. Journal of Experimental Psychology: Human Perception \& Performance, 30, 975-987.

Costen, N. P., Parker, D. M., \& Craw, I. (1994). Spatial content and spatial quantisation effects in face recognition. Perception, 23, 129-146.

Costen, N. P., PARKer, D. M., \& CraW, I. (1996). Effects of high-pass and low-pass spatial filtering on face identification. Perception \& Psychophysics, 58, 602-612.

FIELD, D. J., \& BRADY, N. (1997). Visual sensitivity, blur and the sources of variability in the amplitude spectra of natural scenes. Vision Research, 37, 3367-3383.

Gold, J., Bennett, P. J., \& Sekuler, A. B. (1999). Identification of band-pass filtered letters and faces by human and ideal observers. Vision Research, 39, 3537-3560.

HAYES, A. (1988). Identification of two-tone images; some implications for high- and low-spatial-frequency processes in human vision. Perception, 17, 429-436.

Hayes, T., Morrone, M. C., \& Burr, D. C. (1986). Recognition of positive and negative bandpass-filtered images. Perception, 15, 595-602.

He, S., \& MACLEOD, D. I. (1996). Local luminance nonlinearity and receptor aliasing in the detection of high-frequency gratings. Journal of the Optical Society of America A, 13, 1139-1151.

Kornowski, J. A., \& Petersik, J. T. (2003). Effects on face recognition of spatial-frequency information contained in inspection and test stimuli. Journal of General Psychology, 130, 229-244.

Liu, C. H., Collin, C. A., Rainville, S. J., \& Chaudhuri, A. (2000). The effects of spatial frequency overlap on face recognition. Journal of Experimental Psychology: Human Perception \& Performance, 26, 956-979.

Mauer, D., Le Grand, R., \& Mondloch, C. J. (2002). The many faces of configural processing. Trends in Cognitive Sciences, 6, 255-260.

Morrison, D. J., \& SchYNs, P. G. (2001). Usage of spatial scales for the categorization of faces, objects, and scenes. Psychonomic Bulletin \& Review, 8, 454-469.

NÄSÄNEN, R. (1999). Spatial frequency bandwidth used in the recognition of facial images. Vision Research, 39, 3824-3833.

PARKer, D. M., \& Costen, N. P. (2001). One extreme or the other, or perhaps the golden mean? Issues of spatial resolution in face processing. In H. Ellis \& N. Macrae (Eds.), Validation in psychology: Research perspectives (pp. 151-162). New Brunswick, NJ: Transaction.

Rolls, E. T., BAYlis, G. C., \& Hasselmo, M. E. (1987). The responses of neurons in the cortex in the superior temporal sulcus of the monkey to band-pass spatial frequency filtered faces. Vision Research, 27, 311-326.

Schyns, P. G. (1998). Diagnostic recognition: Task constraints, object information, and their interactions. Cognition, 67, 147-179. 
Schyns, P. G., \& Gosselin, F. (2003). Diagnostic use of scale information for componential and holistic recognition. In M. A. Peterson \& G. Rhodes (Eds.), Perception of faces, objects, and scenes: Analytic and holistic processes (pp. 120-148). Oxford: Oxford University Press.

Tieger, T., \& Ganz, L. (1979). Recognition of faces in the presence of two-dimensional sinusoidal masks. Perception \& Psychophysics, 26, 163-167.

(Manuscript received April 23, 2004;

revision accepted for publication August 26, 2005.) 